\title{
Well posedness results for a class of partial differential equations with hysteresis arising in electromagnetism
}

\author{
Michela Eleuteri ${ }^{1}$
}

\begin{abstract}
We consider an evolutionary P.D.E. motivated by models for electromagnetic processes in ferromagnetic materials. Magnetic hysteresis is represented by means of a hysteresis operator. Under suitable assumptions, an existence and uniqueness theorem is obtained, together with the Lipschitz continuous dependence on the data and some further regularity results. The discussion of the behaviour of the solution in dependence on physical parameters of the problem is also outlined.
\end{abstract}

Classification: 35Q60, 47J40.

Key words: partial differential equations, hysteresis, electromagnetic processes.

\section{Introduction}

The aim of this paper is to study a class of P.D.E.s containing a continuous hysteresis operator $\overline{\mathcal{G}}$, whose model equation can be represented by

$$
\epsilon \frac{\partial^{2} u}{\partial t^{2}}+\sigma \frac{\partial u}{\partial t}-\triangle\left(\gamma \frac{\partial u}{\partial t}+\overline{\mathcal{G}}(u)\right)=\sigma f \quad \text { in } \Omega \times(0, T),
$$

where $\Omega$ is an open bounded set of $\mathbb{R}^{N}, N \geq 1, \epsilon, \sigma$ and $\gamma$ are given positive constants, $\triangle$ is the Laplace operator and $f$ is a given function.

This model equation appears when dealing with electromagnetic processes; in particular it can be obtained by coupling in a suitable way the Maxwell equations, the Ohm law and a constitutive relation between the magnetic field and the magnetic induction. In this context $\epsilon$ is the dielectric permittivity, $\sigma$ is the electric conductivity and $\gamma$ is a relaxation parameter depending on the geometry of the circuit. More precisely, we consider the Ampère and the Faraday laws in a bounded open set of $\mathbb{R}^{3}$, we combine them with the Ohm law and get an equation only involving $\vec{B}$ and $\vec{H}$, the magnetic induction and the magnetic field respectively. The vectorial model obtained is then simplified by imposing some restrictions on the fields; this leads to the scalar character of (1.1). At this point we prescribe a constitutive relation between the magnetic field

\footnotetext{
${ }^{1}$ Università degli Studi di Trento, Dipartimento di Matematica, Via Sommarive 14, I-38050 Povo (Trento), Italy, E-mail: eleuteri@science.unitn.it. Guest of WIAS - Weierstrass-Institute for Applied Analysis and Stochastics, Mohrenstr. 39, D-10117 Berlin, Germany. Partially supported by INdAM and by MIUR - COFIN project "Mathematical modelling and analysis of free boundary problems".
} 
and the magnetic induction. The one we choose can be for example obtained by putting in series a ferromagnetic element with hysteresis and a conducting solenoid filled with a paramagnetic core; the first one is a rate independent element while the second one is a rate dependent one. More about modeling issues is given below in Section 3.

First of all we introduce a weak formulation in Sobolev spaces for a Cauchy problem associated to equation (1.1); under suitable assumptions on the hysteresis operator $\overline{\mathcal{G}}$ and on the data we get existence and uniqueness of the solution of our model problem. The proof of this result is carried on by means of a technique which is based on the contraction mapping principle. Several difficulties arise due the choice of the unusual functional setting: in fact the problem is set within the frame of a non-classical Hilbert triplet

$$
L^{2}(\Omega) \subset H^{-1}(\Omega) \equiv\left(H^{-1}(\Omega)\right)^{\prime} \subset\left(L^{2}(\Omega)\right)^{\prime}
$$

with continuous and dense injections; here the role of the pivot space is played by the Sobolev space $H^{-1}(\Omega)$ endowed with a scalar product chosen ad hoc.

We also obtain the Lipschitz continuous dependence of the solution on the data and some further regularity results.

It is interesting to analyze the behaviour of the solution in dependence of the parameters $\epsilon, \sigma$ and $\gamma$. The case $\epsilon=0$ corresponds to the so called eddy-current approximation, where the displacement current in Ampère's law is neglected; the case $\sigma=0$ means instead that the electric current density $\vec{J}$ vanishes in the whole Euclidean space. Finally the case $\gamma=0$ corresponds to a purely hysteretic constitutive relation, without the presence of rate dependent components of the memory. While the behaviour of the solution as $\epsilon \rightarrow 0$ and $\sigma \rightarrow 0$ can be easily treated, difficulties arise when the parameter $\gamma$ is supposed to vanish. In this case a complete analysis is obtained dealing with a particular case (see [10]).

The Maxwell equations considered in this paper are set in a bounded open set $Q \subset \mathbb{R}^{3}$. However the fields outside this domain may have an influence in the processes which take place inside $Q$. It seems therefore quite difficult to describe this interaction by formulating a boundary value problem on a bounded domain. For this reason we try to describe a more realistic physical approach, where the Ampère law and the Faraday law are taken in the whole $\mathbb{R}^{3}$. Also the Ohm law is extended to a law which holds in the whole Euclidean space; in particular we assume the usual Ohm law inside $Q$ while we suppose that $\vec{J}$ equals a prescribed time-dependent vector field $\vec{J}_{\text {ext }}$ outside $Q$. This is equivalent to assume $\sigma=\chi_{Q}$, where $\chi_{Q}$ is the characteristic function of the set $Q$, so $\sigma$ is no longer a constant but a space parameter dependent function. The vectorial system which comes out from this construction can be also in this case simplified and some particular solutions can be found. For example in [11] we obtain existence and uniqueness of a solution in the one dimensional case, which corresponds to a plane wave. We leave for the moment open the problem to deal with the complete vectorial model.

The paper is organized as follows: after recalling some classical examples of continuous hysteresis operators in Section 2, we analyse the physical interpretation of the model equation (1.1) (Section 3) and present in Section 4 the well posedness results, some of what have been announced in [8] (see also [9], Chapter 2), together with the analysis 
of the behaviour of our solution in dependence of the parameters $\epsilon$ and $\sigma$. Finally Section 5 is devoted to the presentation of a more realistic physical setting, when the electric conductivity is no longer a constant but a space-dependent function.

\section{Hysteresis operators}

\subsection{Hysteresis}

Hysteresis is a phenomenon that appears in several and quite different situations; for example we can encounter it in physics, in engineering, in biology and in many other settings.

According to [26], we can distinguish two main features of hysteresis phenomena: the memory effect and the rate independence.

More precisely, let us consider a system which is described by the couple input-output $(u, w)$. The memory effect means that at any instant $t$ the value of the output is not simply determined by the value $u(t)$ of the input at the same instant but it depends also on the previous evolution of the input $u$. The rate independence instead means that the path of the couple $(u(t), w(t))$ is invariant with respect to any increasing time homeomorphism and so it is independent of its velocity.

A basic contribution to the theory of hysteresis has certainly been brought by Krasnosel'skil and its collaborators. Their work has been summarized in the monograph [13]. In this fundamental work, they introduced the concept of hysteresis operator and started a systematic investigation of its properties. After this pioneering monograph, many scientists, coming also from different areas, contributed to the research on models of hysteresis phenomena; we refer to the recent monographs devoted to this topic of Brokate and Sprekels [5], Krejčí [15], Visintin [26] and also of Bertotti [1], Della Torre [7], Mayergoyz [18] (together with the references therein) for mathematically and physically oriented approaches respectively.

Finally we would like to point out a different approach to hysteresis which has been proposed in the recent years by Mielke, Theil, Levitas and others collaborators; we refer for example to [19], [20], [21]. Their formulation does not involve explicit hysteresis operators, but hysteresis arises implicitly as a result of coupling the energy balance with a stability condition.

\subsection{The play and the stop operators}

Now we briefly recall the definition and some properties of the play and the stop operators, which can be certainly considered the simplest examples of continuous hysteresis operators. 
It is possible to show that (see [15], Section I.3) the following system

(i) $\quad\left|x_{r}(t)\right| \leq r$

$\forall t \in[0, T]$

(ii) $\quad\left(\dot{u}(t)-\dot{x}_{r}(t)\right)\left(x_{r}(t)-\varphi\right) \geq 0$

a.e. $\forall \varphi \in[-r, r]$,

(iii) $\quad x_{r}(0)=x_{r}^{0}$

admits a unique solution $x_{r} \in W^{1,1}(0, T)$ for any given input function $u \in W^{1,1}(0, T)$ and any given initial condition $x_{r}^{0} \in[-r, r]$. Then the stop and the play operators $\mathcal{S}_{r}, \mathcal{P}_{r}:[-r, r] \times W^{1,1}(0, T) \rightarrow W^{1,1}(0, T)$ can be introduced as solution operators of Problem (2.1) by the formula

$$
\mathcal{S}_{r}\left(x_{r}^{0}, u\right):=x_{r} \quad \mathcal{P}_{r}\left(x_{r}^{0}, u\right):=u-x_{r} .
$$

It is also possible to extend these operators to continuous input functions. The set $Z:=[-r, r]$ is called characteristic of the operators $\mathcal{S}_{r}$ and $\mathcal{P}_{r}$. In this case it is a symmetric one-dimensional set but there exist also situations in which more general closed convex sets or tensorial extensions are considered. Other equivalent definitions of the play operator can be found in [5], [13], [16], [17], [26].

Let $\mathcal{P}_{r}$ be the scalar play with characteristic $[-r, r]$. Then for any given input function $u \in W^{1,1}(0, T)$ and any given initial condition $x_{r}^{0} \in[-r, r]$ we have

$$
\mathcal{P}_{r}\left(x_{r}^{0}, u\right)(0):=u(0)-x_{r}^{0} .
$$

We notice that we can associate to any $r \in \mathbb{R}$ the corresponding value $x_{r}^{0}$; this suggests the idea of making the initial configuration of the play system independent of the initial conditions $\left\{x_{r}^{0}\right\}_{r \in \mathbb{R}}$ for the output function by the introduction of some suitable function of $r$. More precisely, following [15] Section II.2, let us consider any function $\lambda \in \Lambda$ where

$$
\Lambda:=\left\{\lambda \in W^{1, \infty}(0, \infty) ;\left|\frac{d \lambda(r)}{d r}\right| \leq 1 \text { a.e. in }[-r, r]\right\} .
$$

We also introduce some useful subspaces of $\Lambda$, i.e.

$$
\Lambda_{R}:=\{\lambda \in \Lambda ; \lambda(r)=0 \text { for } r \geq R\}, \quad \Lambda_{0}:=\bigcup_{R>0} \Lambda_{R} .
$$

$\Lambda$ is called configuration space and the functions $\lambda$ are called memory configurations. If $Q_{r}: \mathbb{R} \rightarrow[-r, r]$ is the projection

$$
Q_{r}(x):=\operatorname{sign}(x) \min \{r,|x|\}=\min \{r, \max \{-r, x\}\},
$$

then we set

$$
x_{r}^{0}:=Q_{r}(u(0)-\lambda(r)) .
$$

This implies that the initial configuration of the play system only depends on $\lambda$ and $u(0)$. The same can be done for the initial configuration $\mathcal{S}_{r}\left(x_{r}^{0}, u\right)(0):=x_{r}^{0}$ of the stop operator. We introduce the following more convenient notation which will be needed later

$$
\wp_{r}(\lambda, u):=\mathcal{P}_{r}\left(x_{r}^{0}, u\right)
$$

for any $\lambda \in \Lambda$, for any $u \in \mathcal{C}^{0}([0, T])$ and $r>0$. 


\subsection{The Preisach operator}

We briefly recall here the definition and some properties of a more complex example of continuous hysteresis operator: the Preisach operator. The construction presented here (which in origin appeared in [14]) can be seen as an alternative to the classical model studied for example in [22] and further investigated in [5], [13], [15], [18], [26] (see also [3], [4], [27], [25]). This approach allows us to obtain a more direct derivation of Theorem 2.4, which plays a central role in our developments.

Let us introduce the Preisach plane, defined as

$$
\mathcal{Q}:=\left\{(r, v) \in \mathbb{R}^{2}: r>0\right\} .
$$

We introduce a function $\psi \in L_{\text {loc }}^{1}(\mathcal{Q})$ such that the following holds

\section{Assumption 2.1.}

there exists $\beta_{1} \in L_{\text {loc }}^{1}(0, \infty)$, such that $0 \leq \psi(r, v) \leq \beta_{1}(r)$ for a.e. $(r, v) \in \mathcal{Q}$.

We put $b_{1}(R):=\int_{0}^{R} \beta_{1}(r) d r$ for $R>0$ and set

$$
g(r, v):=\int_{0}^{v} \psi(r, z) d z \quad \text { for }(r, v) \in \mathcal{Q}
$$

We have the following definition

Definition 2.2. Let $\psi \in L_{\mathrm{loc}}^{1}(\mathcal{Q})$ be given and let $g$ be chosen as in (2.4). Then the Preisach operator $\mathcal{W}: \Lambda_{0} \times \mathcal{C}^{0}([0, T]) \rightarrow \mathcal{C}^{0}([0, T])$ generated by the function $g$ is defined by the formula

$$
\mathcal{W}[\lambda, u](t):=\int_{0}^{\infty} g\left(r, \wp_{r}[\lambda, u](t)\right) d r
$$

for any given $\lambda \in \Lambda_{0}, u \in \mathcal{C}^{0}([0, T])$ and $t \in[0, T]$, where $\Lambda_{0}$ is introduced in (2.3).

Then we can state the following result (see [15], Section II.3, Proposition 3.11)

Proposition 2.3. Let Assumption 2.1 be satisfied and let $R>0$ be given. Then $\mathcal{W}$ is a hysteresis operator and for every $\lambda, \mu \in \Lambda_{R}$ and $u, v \in \mathcal{C}^{0}([0, T])$ such that $\|u\|_{\mathcal{C}^{0}([0, T])},\|v\|_{\mathcal{C}^{0}([0, T])} \leq R$, it satisfies

$$
\|\mathcal{W}[\lambda, u]-\mathcal{W}[\mu, v]\|_{\mathcal{C}^{0}([0, T])} \leq \int_{0}^{R}|\lambda(r)-\mu(r)| \beta_{1}(r) d r+b_{1}(R)\|u-v\|_{\mathcal{C}^{0}([0, T])} .
$$

The following important result which will play a central role in the following (see [15], Section II.3, Theorem 3.17, see also [6], Theorem 5.8), holds

Theorem 2.4. Let Assumption 2.1 be fulfilled and let $\lambda \in \Lambda_{0}, b>0$ be given. Then the operator $b I+\mathcal{W}(\lambda, \cdot): \mathcal{C}^{0}([0, T]) \rightarrow \mathcal{C}^{0}([0, T])$, where $I$ is the identity operator and $\mathcal{W}$ is the Preisach operator introduced in (2.5), is invertible and its inverse is Lipschitz continuous. 
As a straightforward application of this theorem, let us fix any $\lambda \in \Lambda$ and introduce the operator

$$
\mathcal{F}: \mathcal{C}^{0}([0, T]) \rightarrow \mathcal{C}^{0}([0, T]) \quad \mathcal{F}(v)(t)=v(t)+4 \pi \mathcal{W}[\lambda, v](t),
$$

where $\mathcal{W}[\lambda, \cdot]$ is defined in $(2.5)$ (the introduction of the operator $\mathcal{F}$ will become clear in Section 3, where the physical context in which our model problem arises will be outlined).

Now Theorem 2.4 gives us that $\mathcal{F}$ is invertible and its inverse is a Lipschitz continuous operator. Let us set $\mathcal{G}=\mathcal{F}^{-1}$ and denote with $L_{\mathcal{G}}$ the Lipschitz constant of the operator $\mathcal{G}$. It can be easily shown that also $\mathcal{G}$ is a hysteresis operator.

\subsection{Parameter dependent hysteresis}

Operators like $\mathcal{W}[\lambda, \cdot]$ in $(2.5)$ can be used to model phenomena in which time is the only independent variable like in O.D.E.s. In the case of P.D.E.s, when also the space variable appears, it is necessary to extend the construction of the Preisach model outlined so far. More precisely, we have to consider both the input and the initial memory configuration $\lambda$ that additionally depend on the space parameter $x$.

Thus, from now on, let us fix any initial memory configuration

$$
\lambda \in L^{2}\left(\Omega ; \Lambda_{R}\right), \quad \text { for some } R>0,
$$

where $\Lambda_{R}$ has been introduced in (2.3). Let $\mathcal{M}\left(\Omega ; \mathcal{C}^{0}([0, T])\right)$ be the Fréchet space of strongly measurable functions $\Omega \rightarrow \mathcal{C}^{0}([0, T])$, i.e. the space of functions $v: \Omega \rightarrow$ $\mathcal{C}^{0}([0, T])$ such that there exists a sequence $v_{n}$ of simple functions with $v_{n} \rightarrow v$ strongly in $\mathcal{C}^{0}([0, T])$ a.e. in $\Omega$.

Now let us set

$$
\begin{array}{ll}
\overline{\mathcal{F}}: \mathcal{M}\left(\Omega ; \mathcal{C}^{0}([0, T])\right) \rightarrow \mathcal{M}\left(\Omega ; \mathcal{C}^{0}([0, T])\right) & \overline{\mathcal{F}}(u)(x, t):=\mathcal{F}(u(x, \cdot))(t) \\
\overline{\mathcal{G}}: \mathcal{M}\left(\Omega ; \mathcal{C}^{0}([0, T])\right) \rightarrow \mathcal{M}\left(\Omega ; \mathcal{C}^{0}([0, T])\right) & \overline{\mathcal{G}}:=\overline{\mathcal{F}}^{-1}
\end{array}
$$

where $\mathcal{F}$ has been introduced in (2.6).

It turns out that

$$
\overline{\mathcal{G}}(w)(x, t):=\mathcal{G}(w(x, \cdot))(t) \quad \forall w \in \mathcal{M}\left(\Omega ; \mathcal{C}^{0}([0, T])\right) ;
$$

moreover, as $\mathcal{G}$ is a Lipschitz continuous hysteresis operator with Lipschitz constant $L_{\mathcal{G}}$, we have that $\overline{\mathcal{G}}$ is also a hysteresis operator and in addition $\overline{\mathcal{G}}$ is:

- Lipschitz continuous with Lipschitz constant $L_{\mathcal{G}}$, in the following sense: for any $u_{1}, u_{2} \in \mathcal{M}\left(\Omega ; \mathcal{C}^{0}([0, T])\right)$, a.e in $\Omega$

$$
\left\|\left[\overline{\mathcal{G}}\left(u_{1}\right)\right](x, \cdot)-\left[\overline{\mathcal{G}}\left(u_{2}\right)\right](x, \cdot)\right\|_{\mathcal{C}^{0}([0, T])} \leq L_{\mathcal{G}}\left\|u_{1}(x, \cdot)-u_{2}(x, \cdot)\right\|_{\mathcal{C}^{0}([0, T])} ;
$$

- gradient bounded in the following sense: for all $u \in \mathcal{M}\left(\Omega ; \mathcal{C}^{0}([0, T])\right)$, for a.e. $x, y \in$ $\Omega$,

$$
\|[\overline{\mathcal{G}}(u)](x, \cdot)-[\overline{\mathcal{G}}(u)](y, \cdot)\|_{\mathcal{C}^{0}([0, T])} \leq L_{\mathcal{G}}\|u(x, \cdot)-u(y, \cdot)\|_{\mathcal{C}^{0}([0, T])} .
$$




\section{Physical interpretation of the model equation (1.1)}

Electromagnetic processes in ferromagnetic materials can be described by coupling in a suitable way the Maxwell equations with the Ohm law. Here we consider $Q \subset \mathbb{R}^{3}$ to be an electromagnetic material; we set $Q_{T}:=Q \times(0, T)$, for a fixed $T>0$ and we recall the Ampère, Faraday and Ohm laws (where $\nabla \times$ is the curl operator)

$$
\begin{array}{ll}
c \nabla \times \vec{H}=4 \pi \vec{J}+\frac{\partial \vec{D}}{\partial t} & \text { in } Q_{T} \\
c \nabla \times \vec{E}=-\frac{\partial \vec{B}}{\partial t} & \text { in } Q_{T} \\
\vec{J}=\sigma(\vec{E}+\vec{g}) & \text { in } Q_{T}
\end{array}
$$

where $c$ is the speed of light in vacuum, $\vec{H}$ is the magnetic field, $\vec{J}$ is the electric current density, $\vec{D}$ is the electric displacement, $\vec{E}$ is the electric field, $\vec{B}$ is the magnetic induction, $\sigma$ is the electric conductivity and finally $\vec{g}$ is an applied electromotive force. For more details about these facts see a classical text of electromagnetism, for example $[12]$.

We assume for simplicity that $\vec{D}=\epsilon \vec{E}$, where $\epsilon$ is the dielectric permittivity. Applying the curl operator to (3.1), differentiating (3.2) in time and eliminating $\vec{J}, \vec{D}$ and $\vec{E}$ we then get

$$
\epsilon \frac{\partial^{2} \vec{B}}{\partial t^{2}}+4 \pi \sigma \frac{\partial \vec{B}}{\partial t}+c^{2} \nabla \times \nabla \times \vec{H}=4 \pi c \sigma \nabla \times \vec{g} \quad \text { in } Q_{T}
$$

We further simplify equation (3.4) by considering planar waves only. More precisely, let $\Omega$ be a domain of $\mathbb{R}^{2}$, we set $\Omega_{T}:=\Omega \times(0, T)$ and assume that (using orthogonal Cartesian coordinates $x, y, z)$ both $\vec{B}$ and $\vec{H}$ are parallel to the $z$-axis and only depend on the coordinates $x, y$, i.e. $\vec{B}=(0,0, B(x, y))$ and $\vec{H}=(0,0, H(x, y))$. Then

$$
\nabla \times \nabla \times \vec{H}=\left(0,0,-\triangle_{x, y} H\right) \quad\left(\triangle_{x, y}:=\frac{\partial^{2}}{\partial x^{2}}+\frac{\partial^{2}}{\partial y^{2}}\right) \quad \text { in } \Omega_{T} .
$$

If moreover $\nabla \times \vec{g}:=(0,0, g)$, equation (3.4) is then reduced to an equation for scalar variables

$$
\epsilon \frac{\partial^{2} B}{\partial t^{2}}+4 \pi \sigma \frac{\partial B}{\partial t}-c^{2} \triangle_{x, y} H=4 \pi c \sigma g=\sigma f \quad \text { in } \Omega_{T},
$$

where we set $f:=4 \pi c g$.

At this point we would like to combine equation (3.5) with the constitutive relation

$$
H=\overline{\mathcal{G}}(B)+\gamma \frac{\partial B}{\partial t}
$$

where $\overline{\mathcal{G}}$ is a suitable hysteresis operator and $\gamma>0$ is a given constant. The relation (3.6) can be for example obtained by the combination in series of a ferromagnetic element with hysteresis and a conducting solenoid filled with a paramagnetic material. 
We model a ferromagnetic element with hysteresis by assuming that $B=H+4 \pi M$ with a hysteresis dependence of the magnetization $M$ on $H$, i.e. $M=\overline{\mathcal{W}}(H)$ where $\overline{\mathcal{W}}$ is a scalar Preisach operator. This means that $B=H+4 \pi \overline{\mathcal{W}}(H)=: \overline{\mathcal{F}}(H)$. Providing that the inverse of the operator $\overline{\mathcal{F}}$ exists we may then assume that

$$
H=\overline{\mathcal{F}}^{-1}(B)=: \overline{\mathcal{G}}(B) .
$$

This justifies the choice of the operators (2.6) and (2.8).

In the case of the conducting solenoid having a paramagnetic core, the equation

$$
H=\gamma \frac{\partial B}{\partial t}
$$

describes the so called linear induction; this equation can be justified as follows: a flux variation $\frac{\partial B}{\partial t}$ induces the magnetic field

$$
\tilde{H}=-\gamma \frac{\partial B}{\partial t}
$$

and this can be seen using the Faraday-Lenz and the Ampère laws (the constant $\gamma>0$ depends on the geometry of the circuit). Hence to vary the flux, the opposite magnetic field must be applied $H=-\tilde{H}$ and this leads to equation (3.7). So in (3.6) we have the presence of a rate independent element and a rate dependent one.

Now we consider the equation which results from the combination of (3.5) with (3.6). We write it only displaying the coefficients $\epsilon, \sigma$ and $\gamma$ which are the only ones of some importance in our development. We get

$$
\epsilon \frac{\partial^{2} B}{\partial t^{2}}+\sigma \frac{\partial B}{\partial t}-\triangle_{x, y}\left(\gamma \frac{\partial B}{\partial t}+\overline{\mathcal{G}}(B)\right)=\sigma f
$$

i.e. equation (1.1).

\section{Well posedness results for model equation (1.1)}

\subsection{Choice of the functional setting}

We fix an open bounded set $\Omega \subset \mathbb{R}^{N}, N \geq 1$ of Lipschitz class with boundary $\Gamma$ and set $\Omega_{T}:=\Omega \times(0, T)$.

In this section we are going to discuss the setting of our model problem. The choice of the right functional spaces to work with plays a fundamental role in order to have some positive results. Let us explain a bit in detail our choice of functional framework which will be relevant for the right interpretation of the weak formulation of our problem.

Let $H^{-1}(\Omega)$ be the dual of $H_{0}^{1}(\Omega)$. First of all we consider the injection of the space $L^{2}(\Omega)$ into the space $H^{-1}(\Omega)$. More precisely we take the map $j: L^{2}(\Omega) \rightarrow H^{-1}(\Omega)$ which acts in the following way

$$
H^{-1}(\Omega)\langle j(\phi), \varphi\rangle_{H_{0}^{1}(\Omega)}:=\int_{\Omega} \phi \varphi d x \quad \forall \phi \in L^{2}(\Omega), \forall \varphi \in H_{0}^{1}(\Omega) .
$$


It is not difficult to see that $j$ is a continuous and dense injection, i.e. $L^{2}(\Omega)$ is a linear subspace of $H^{-1}(\Omega)$ and it is dense with respect to the strong topology of $H^{-1}(\Omega)$. Then we may identify the space $\left(H^{-1}(\Omega)\right)^{\prime}$ with a linear subspace of $\left(L^{2}(\Omega)\right)^{\prime}$, i.e. $\left(H^{-1}(\Omega)\right)^{\prime} \subset\left(L^{2}(\Omega)\right)^{\prime}$ with continuous injection (let us call this map $j^{*}$ ). More precisely we identify functionals with their restrictions, i.e.

$$
\left(L^{2}(\Omega)\right)^{\prime}\left\langle j^{*} \psi, \phi\right\rangle_{L^{2}(\Omega)}:={ }_{\left(H^{-1}(\Omega)\right)^{\prime}}\langle\psi, j(\phi)\rangle_{H^{-1}(\Omega)} \quad \forall \psi \in\left(H^{-1}(\Omega)\right)^{\prime}, \forall \phi \in L^{2}(\Omega) .
$$

In the following we will avoid to write each time $j, j^{*}$ when it will be clear from the context, in order to simplify the notations. So for example (4.2) will simply become

$$
\left(L^{2}(\Omega)\right)^{\prime}\langle\psi, \phi\rangle_{L^{2}(\Omega)}:={ }_{\left(H^{-1}(\Omega)\right)^{\prime}}\langle\psi, \phi\rangle_{H^{-1}(\Omega)}, \quad \forall \psi \in\left(H^{-1}(\Omega)\right)^{\prime}, \forall \phi \in L^{2}(\Omega) .
$$

Now we introduce the operator $A: H_{0}^{1}(\Omega) \rightarrow H^{-1}(\Omega)$ defined as follows

$$
H^{-1}(\Omega)\langle A u, v\rangle_{H_{0}^{1}(\Omega)}:=\int_{\Omega} \nabla u \cdot \nabla v d x \quad \forall u, v \in H_{0}^{1}(\Omega) ;
$$

so it is clear that $A u=-\triangle u\left(:=-\sum_{i=1}^{N} \frac{\partial^{2} u}{\partial x_{i}^{2}}\right)$ in the sense of distributions.

In this setting we can think to invert the Laplace operator, i.e. the operator $A^{-1}$ can be interpreted as the inverse of the operator $-\triangle$ associated with the homogeneous Dirichlet boundary conditions. More precisely, for any $v \in H^{-1}(\Omega)$,

$$
u=A^{-1} v \text { if and only if }\left\{\begin{array}{l}
u \in H^{1}(\Omega) \\
-\triangle u=v \text { in } \mathcal{D}^{\prime}(\Omega) \\
\gamma_{0} u=0 \text { on } \partial \Omega=\Gamma
\end{array}\right.
$$

where $\gamma_{0}: H^{1}(\Omega) \rightarrow H^{1 / 2}(\Gamma)$ is the unique linear continuous trace operator such that

$$
\gamma_{0} v=v_{\left.\right|_{\Gamma}} \quad \forall v \in \mathcal{C}^{\infty}(\bar{\Omega}) \cap H^{1}(\Omega) .
$$

At this point we consider the space $H^{-1}(\Omega)$ endowed with the scalar product

$$
(u, v)_{H^{-1}(\Omega)}:=_{H^{-1}(\Omega)}<u, A^{-1} v>_{H_{0}^{1}(\Omega)} .
$$

It is clear, using (4.1), that

$$
(u, v)_{H^{-1}(\Omega)}:=\int_{\Omega} u A^{-1} v d x \quad \forall u \in L^{2}(\Omega) .
$$

Finally we identify the space $H^{-1}(\Omega)$ with its dual by means of the Riesz operator $\mathcal{R}: H^{-1}(\Omega) \rightarrow\left(H^{-1}(\Omega)\right)^{\prime}$ which acts in the following way

$$
\left(H^{-1}(\Omega)\right)^{\prime}\langle\mathcal{R} u, v\rangle_{H^{-1}(\Omega)}:=(u, v)_{H^{-1}(\Omega)} \quad \forall u, v \in H^{-1}(\Omega) .
$$

Let us remark that with this identification we immediately get, (omitting from now on also the Riesz operator $\mathcal{R}$ for the sake of simplicity),

$$
\begin{aligned}
& \left(L^{2}(\Omega)\right)^{\prime}\langle\psi, \phi\rangle_{L^{2}(\Omega)} \stackrel{(4.2)(4.5)}{=}(\psi, \phi)_{H^{-1}(\Omega)}=(\phi, \psi)_{H^{-1}(\Omega)} \stackrel{(4.4)}{=}_{H^{-1}(\Omega)}\left\langle\phi, A^{-1} \psi\right\rangle_{H_{0}^{1}(\Omega)} \\
& \stackrel{(4.1)}{=} \int_{\Omega} \phi A^{-1} \psi d x \quad \forall \phi \in L^{2}(\Omega), \quad \forall \psi \in H^{-1}(\Omega),
\end{aligned}
$$


where we also used the fact that the scalar product $(\cdot, \cdot)_{H^{-1}(\Omega)}$ is symmetric.

As $L^{2}(\Omega) \subset H^{-1}(\Omega)$ with continuous and dense injection, we then have the Hilbert triplet

$$
L^{2}(\Omega) \subset H^{-1}(\Omega) \equiv\left(H^{-1}(\Omega)\right)^{\prime} \subset\left(L^{2}(\Omega)\right)^{\prime}
$$

with continuous and dense injections.

\subsection{Weak formulation of the model problem}

For the sake of simplicity, from now on we set $L^{2}(\Omega):=V, H^{-1}(\Omega):=H$ and $\left(L^{2}(\Omega)\right)^{\prime}:=V^{\prime}$. We suppose that $u^{0} \in V, v^{0} \in H$ and $f \in L^{2}(0, T ; H)$ are given; we also assume that $\epsilon, \sigma$ and $\gamma$ are given positive constants. Let us consider an initial memory configuration $\lambda$ as in (2.7) and let $\overline{\mathcal{G}}$ be the hysteresis operator introduced in (2.8). We want to solve the following problem.

Problem 4.1. We search for two functions $u \in \mathcal{M}\left(\Omega ; \mathcal{C}^{0}([0, T])\right) \cap H^{1}(0, T ; V)$ and $v \in L^{2}\left(\Omega_{T}\right)$ such that $\overline{\mathcal{G}}(u) \in L^{2}\left(\Omega_{T}\right)$ and for any $\psi \in H^{1}(0, T ; V)$ with $\psi(\cdot, T)=0$ a.e. in $\Omega$

$$
\begin{aligned}
\int_{0}^{T}-V_{V^{\prime}}\left\langle\epsilon v+\sigma u, \frac{\partial \psi}{\partial t}\right\rangle_{V} d t & +\int_{0}^{T} \int_{\Omega}(\gamma v+\overline{\mathcal{G}}(u)) \psi d x d t=\sigma \int_{0}^{T} V^{\prime}\langle f, \psi\rangle_{V} d t \\
& +V_{V^{\prime}}\left\langle\left(\epsilon v^{0}+\sigma u^{0}\right)(\cdot), \psi(\cdot, 0)\right\rangle_{V} \\
-\int_{0}^{T} \int_{\Omega} u \frac{\partial \psi}{\partial t} d x d t & =\int_{0}^{T} \int_{\Omega} v \psi d x d t+\int_{\Omega} u^{0}(\cdot) \psi(\cdot, 0) d x
\end{aligned}
$$

Interpretation. Following [24], we first introduce, for any given Banach space $X$, the space of distributions $\mathcal{D}^{\prime}(0, T ; X)$ on $(0, T)$ taking values in $X$ which is defined as $\mathcal{D}^{\prime}(0, T ; X):=\mathcal{L}(\mathcal{D}(0, T) ; X)$, where $\mathcal{L}(Y, Z)$ is the space of linear and continuous mappings from $Y$ to $Z$.

If $f \in \mathcal{D}^{\prime}(0, T ; X)$ one can define its distributional derivative in the following way

$$
\frac{\partial f}{\partial t}(\varphi)=-f \frac{\partial \varphi}{\partial t} \quad \forall \varphi \in \mathcal{D}(0, T) .
$$

For a function $f \in L^{p}(0, T ; X)$ one can introduce a distribution (still denoted by $f$ ) on $(0, T)$ taking values in $X$ in the following way

$$
f(\varphi)=\int_{0}^{T} f(t) \varphi(t) d t \quad \varphi \in \mathcal{D}(0, T)
$$

this integral is an element of $X$. Also in this case we can define $\frac{\partial f}{\partial t}$ as an element of $\mathcal{D}^{\prime}(0, T ; X)$ using (4.8). For more details on the definition of derivatives taking values in a Banach space and related topics, see for example [24].

After this preamble, let us show that (4.6) implies

$$
A^{-1}\left(\epsilon \frac{\partial v}{\partial t}+\sigma \frac{\partial u}{\partial t}\right)+\gamma v+\overline{\mathcal{G}}(u)=\sigma A^{-1} f \quad \text { in } \mathcal{D}^{\prime}\left(0, T ; L^{2}(\Omega)\right) .
$$


In fact, let us take any $\varphi \in \mathcal{D}(0, T)$ and any $\phi \in L^{2}(\Omega)$. Consider $\psi \in \mathcal{D}\left(0, T ; L^{2}(\Omega)\right)$ defined in the following way

$$
\psi(t, x):=\varphi(t) \phi(x) .
$$

It is easy to see that in particular $\psi \in H^{1}(0, T ; V)$ and $\psi(\cdot, T)=0$ a.e. in $\Omega$, so (4.6) holds for this particular choice of $\psi$. Taking into account that $\frac{\partial \varphi}{\partial t}(t) \in \mathbb{R}$ for any $t \in[0, T]$, we have

$$
\begin{aligned}
& \int_{0}^{T}-V_{V^{\prime}}\left\langle\epsilon v(t)+\sigma u(t), \frac{\partial \psi}{\partial t}(t)\right\rangle_{V} d t \\
& \stackrel{(4.2)(4.5)}{=} \int_{0}^{T}-\left(\epsilon v(t)+\sigma u(t), \frac{\partial \varphi}{\partial t}(t) \phi\right)_{H^{-1}(\Omega)} d t \\
& \stackrel{(4.4)}{=} \int_{0}^{T}-_{H^{-1}(\Omega)}\left\langle\phi, \frac{\partial \varphi}{\partial t}(t) A^{-1}(\epsilon v(t)+\sigma u(t))\right\rangle_{H_{0}^{1}(\Omega)} d t \\
& \stackrel{(4.1)}{=}-\int_{0}^{T} \int_{\Omega} \frac{\partial \varphi}{\partial t} A^{-1}(\epsilon v+\sigma u) \phi d x d t,
\end{aligned}
$$

where we also used the fact the scalar product of $H^{-1}(\Omega)$ is symmetric and the fact that $\phi$ does not depend on $t$. The same can be done with the term $\sigma \int_{0}^{T} V^{\prime}\langle f, \psi\rangle_{V} d t$ so that we obtain for all $\phi \in L^{2}(\Omega)$

$$
\begin{aligned}
& \int_{\Omega}\left[\int _ { 0 } ^ { T } \left\{-A^{-1}(\epsilon v(t)+\sigma u(t)) \frac{\partial \varphi}{\partial t}(t)+(\gamma v(t)+\overline{\mathcal{G}}(u)(t)) \varphi(t)\right.\right. \\
& \left.\left.-\sigma A^{-1} f(t) \varphi(t)\right\} d t\right] \phi d x=0
\end{aligned}
$$

let us notice that with our choice of $\psi$ the term ${ }_{V^{\prime}}\left\langle\left(\epsilon v^{0}+\sigma u^{0}\right)(\cdot), \psi(\cdot, 0)\right\rangle_{V}$ vanishes as we have $\psi(\cdot, 0)=0$ a.e. in $\Omega$. The previous equation is equivalent to

$$
\begin{aligned}
& \int_{0}^{T}\left\{-A^{-1}(\epsilon v(t)+\sigma u(t)) \frac{\partial \varphi}{\partial t}(t)+(\gamma v(t)+\overline{\mathcal{G}}(u)(t)) \varphi(t)\right. \\
& \left.-\sigma A^{-1} f(t) \varphi(t)\right\} d t=0
\end{aligned}
$$

in $L^{2}(\Omega)$. Using (4.8) and (4.9), we easily obtain (4.10), so at the end (4.6) and (4.7) yield

$$
\left\{\begin{array}{l}
A^{-1} \epsilon \frac{\partial v}{\partial t}+A^{-1} \sigma \frac{\partial u}{\partial t}+\gamma v+\overline{\mathcal{G}}(u)=\sigma A^{-1} f \\
\frac{\partial u}{\partial t}=v
\end{array} \quad \text { in } \mathcal{D}^{\prime}\left(0, T ; L^{2}(\Omega)\right) .\right.
$$

At this point, by comparison we have that

$$
\epsilon A^{-1} \frac{\partial v}{\partial t} \in L^{2}\left(\Omega_{T}\right)
$$


thus (4.11) holds in $L^{2}(\Omega)$ a.e. in $(0, T)$. Therefore $A^{-1} v \in H^{1}\left(0, T ; L^{2}(\Omega)\right)$ and, integrating by parts in (4.6) and (4.7), we obtain

$$
A^{-1} u_{\left.\right|_{t=0}}=A^{-1} u^{0} \quad A^{-1} v_{\left.\right|_{t=0}}=A^{-1} v^{0} \text { in } L^{2}(\Omega), \text { in the sense of traces. }
$$

In turn, (4.11) and (4.12) yield (4.6) and (4.7) and the two formulations are equivalent. We end this part by noticing that, if in addition the solution $(u, v)$ is more regular in space, (as indeed happens at the end in Theorem 4.5), then (4.6) and (4.7) can be also interpreted as

$$
\left\{\begin{array}{l}
\epsilon \frac{\partial v}{\partial t}+\sigma \frac{\partial u}{\partial t}-\triangle(\gamma v+\overline{\mathcal{G}}(u))=\sigma f \\
\frac{\partial u}{\partial t}=v
\end{array} \quad \text { in } L^{2}\left(0, T ; V^{\prime}\right)\right.
$$

and so we come back to the original model equation from what our discussion started.

\subsection{An existence and uniqueness result}

Theorem 4.2. (Existence and uniqueness).

Let $\overline{\mathcal{G}}: \mathcal{M}\left(\Omega ; \mathcal{C}^{0}([0, T])\right) \rightarrow \mathcal{M}\left(\Omega ; \mathcal{C}^{0}([0, T])\right)$ be the operator introduced in (2.8). Suppose that

$$
u^{0} \in V, \quad v^{0} \in H, \quad f \in L^{2}(0, T ; H) .
$$

Then Problem 4.1 has a unique solution

$$
u \in H^{1}\left(0, T ; L^{2}(\Omega)\right), \quad v \in L^{2}\left(\Omega_{T}\right)
$$

such that

$$
\overline{\mathcal{G}}(u) \in H^{1}\left(0, T ; L^{2}(\Omega)\right) .
$$

Proof. The proof of this theorem consists in two steps.

- Step 1: Auxiliary Problem. In this first step we fix $z \in H^{1}(0, T ; V)$, where we recall that we set, for brevity, $V:=L^{2}(\Omega)$, and we consider Problem $4.1_{Z}$ which is the auxiliary problem obtained from Problem 4.1 by replacing $\overline{\mathcal{G}}(u)$ with $\overline{\mathcal{G}}(z)$. We can interpret Problem $4.1_{Z}$ as

$$
\left\{\begin{array}{l}
\epsilon A^{-1} \frac{\partial v}{\partial t}+\sigma A^{-1} \frac{\partial u}{\partial t}+\gamma v=\sigma A^{-1} f-\overline{\mathcal{G}}(z)=\sigma A^{-1}(f-A \overline{\mathcal{G}}(z))=: A^{-1} G_{z} \\
\frac{\partial u}{\partial t}=v
\end{array}\right.
$$

which holds in $\mathcal{D}^{\prime}\left(0, T ; L^{2}(\Omega)\right)$, together with the initial values

$$
A^{-1} u_{\left.\right|_{t=0}}=A^{-1} u^{0} \quad A^{-1} v_{\mid t=0}=A^{-1} v^{0} \quad \text { in } L^{2}(\Omega), \text { in the sense of traces. }
$$

By comparison, the previous system actually holds in $L^{2}\left(\Omega_{T}\right)$ and then in $L^{2}(\Omega)$ a.e. in $(0, T)$. So in order to prove that this problem admits a unique solution $(u, v)$, it is 
enough to show that there exists a unique $v$ such that, for all $\phi \in L^{2}(\Omega)$ and for a.e. $t \in[0, T]$,

$$
\int_{\Omega} \phi A^{-1} \epsilon \frac{\partial v}{\partial t} d x+\int_{\Omega}\left(A^{-1} \sigma v+\gamma v\right) \phi d x=\int_{\Omega} \phi A^{-1} G_{z} d x
$$

with the initial data (4.13). This can be done using a classical theorem for evolution equations of parabolic type, such as for example, Theorem X.9 contained in Chapter $\mathrm{X}$ of [2]. This theorem also gives the following regularity for $v$

$$
v \in L^{2}\left(\Omega_{T}\right) \cap \mathcal{C}^{0}\left(0, T ; H^{-1}(\Omega)\right), \quad \frac{\partial v}{\partial t} \in L^{2}\left(0, T ;\left(L^{2}(\Omega)\right)^{\prime}\right)
$$

- SECOND STEP: FIXED POINT. At this point, we introduce the set

$$
B=\left\{z \in H^{1}(0, T ; V): z(0)=u^{0}\right\} .
$$

For each $z \in B$ we found, in the previous step, a unique solution $(u, v)$ of Problem $4.1_{Z}$. Thus we may construct an operator

$$
J: B \rightarrow B \quad z \mapsto u .
$$

Now we consider a couple of data $z_{1}, z_{2} \in B$; let us define $u_{1}:=J\left(z_{1}\right), u_{2}:=J\left(z_{2}\right)$. If $v_{i}:=\frac{\partial u_{i}}{\partial t}$ for $i=1,2$, then we have the following equation

$$
\epsilon A^{-1} \frac{\partial}{\partial t}\left(v_{1}-v_{2}\right)+\sigma A^{-1} \frac{\partial}{\partial t}\left(u_{1}-u_{2}\right)+\gamma\left(v_{1}-v_{2}\right)+\overline{\mathcal{G}}\left(z_{1}\right)-\overline{\mathcal{G}}\left(z_{2}\right)=0,
$$

which holds in $L^{2}\left(\Omega_{T}\right)$. Let us multiply $(4.15)$ by $\left(v_{1}-v_{2}\right)$ in the scalar product of $L^{2}(\Omega)$. We have

$$
\begin{aligned}
& \epsilon \int_{\Omega}\left(A^{-1} \frac{\partial}{\partial t}\left(v_{1}-v_{2}\right)\right)\left(v_{1}-v_{2}\right) d x+\sigma \int_{\Omega}\left(A^{-1} \frac{\partial}{\partial t}\left(u_{1}-u_{2}\right)\right)\left(v_{1}-v_{2}\right) d x \\
& +\gamma \int_{\Omega}\left|v_{1}-v_{2}\right|^{2} d x+\int_{\Omega}\left(\overline{\mathcal{G}}\left(z_{1}\right)-\overline{\mathcal{G}}\left(z_{2}\right)\right)\left(v_{1}-v_{2}\right) d x \\
= & \epsilon\left(\frac{\partial}{\partial t}\left(v_{1}-v_{2}\right), v_{1}-v_{2}\right)_{H}(t)+\sigma\left\|v_{1}-v_{2}\right\|_{H}^{2}(t)+\gamma \int_{\Omega}\left|v_{1}-v_{2}\right|^{2} d x \\
& +\int_{\Omega}\left(\overline{\mathcal{G}}\left(z_{1}\right)-\overline{\mathcal{G}}\left(z_{2}\right)\right)\left(v_{1}-v_{2}\right) d x=\epsilon \frac{1}{2} \frac{d}{d t}\left\|v_{1}-v_{2}\right\|_{H}^{2}(t) \\
& +\sigma\left\|v_{1}-v_{2}\right\|_{H}^{2}(t)+\gamma\left\|v_{1}-v_{2}\right\|_{L^{2}(\Omega)}^{2}(t)+\int_{\Omega}\left(\overline{\mathcal{G}}\left(z_{1}\right)-\overline{\mathcal{G}}\left(z_{2}\right)\right)\left(v_{1}-v_{2}\right) d x=0 .
\end{aligned}
$$

Now, setting $D(t)=\frac{1}{2}\left\|v_{1}-v_{2}\right\|_{H}^{2}(t)$, we deduce

$$
\epsilon \frac{d D}{d t}(t)+\gamma\left\|v_{1}-v_{2}\right\|_{L^{2}(\Omega)}^{2}(t) \leq \frac{1}{\gamma}\left\|\overline{\mathcal{G}}\left(z_{1}\right)-\overline{\mathcal{G}}\left(z_{2}\right)\right\|_{L^{2}(\Omega)}^{2}(t)+\frac{\gamma}{4}\left\|v_{1}-v_{2}\right\|_{L^{2}(\Omega)}^{2}(t)
$$


On the other hand

$$
\begin{aligned}
\int_{\Omega}\left[\overline{\mathcal{G}}\left(z_{1}\right)(x, t)-\overline{\mathcal{G}}\left(z_{2}\right)(x, t)\right]^{2} d x & \stackrel{(2.9)}{\leq} L_{\mathcal{G}}^{2} \int_{\Omega}\left\|z_{1}(x, \cdot)-z_{2}(x, \cdot)\right\|_{\mathcal{C}^{0}([0, t])}^{2} d x \\
& \leq L_{\mathcal{G}}^{2} \int_{\Omega}\left(\int_{0}^{t}\left|\frac{\partial}{\partial \tau}\left(z_{1}-z_{2}\right)\right|(x, \tau) d \tau\right)^{2} d x \\
& \leq L_{\mathcal{G}}^{2} t \int_{0}^{t} \int_{\Omega}\left|\frac{\partial}{\partial \tau}\left(z_{1}-z_{2}\right)\right|^{2}(x, \tau) d x d \tau
\end{aligned}
$$

where we used the fact that $z_{1}(0)=z_{2}(0)$, as $z_{1}, z_{2} \in B$.

We introduce an equivalent norm on $H^{1}(0, T ; V)$, namely

$$
\|z\|:=\left(\|z(0)\|_{L^{2}(\Omega)}^{2}+\int_{0}^{T} \exp \left(-\frac{2 L_{\mathcal{G}}^{2} t^{2}}{\gamma^{2}}\right)\left\|\frac{\partial z}{\partial t}\right\|_{L^{2}(\Omega)}^{2}(t) d t\right)^{1 / 2} \quad \forall z \in H^{1}(0, T ; V) .
$$

At this point we multiply (4.16) by $\exp \left(-\frac{2 L_{\mathcal{G}}^{2} t^{2}}{\gamma^{2}}\right)$ and integrate in time, for $t \in$ $(0, T)$. We use (4.17) and the fact that $v_{1}(x, 0)=v_{2}(x, 0)$ (this makes sense since, from the previous step we have $\left.v \in \mathcal{C}^{0}\left(0, T ; H^{-1}(\Omega)\right)\right)$. So we deduce

$$
\begin{aligned}
\frac{3}{4}\left\||| J\left(z_{1}\right)-J\left(z_{2}\right) \mid\right\|^{2} \leq & \int_{0}^{T} \exp \left(-\frac{2 L_{\mathcal{G}}^{2} t^{2}}{\gamma^{2}}\right) \frac{L_{\mathcal{G}}^{2} t}{\gamma^{2}} \int_{0}^{t} \int_{\Omega}\left|\frac{\partial}{\partial \tau}\left(z_{1}-z_{2}\right)\right|^{2}(x, \tau) d x d \tau \\
= & -\frac{1}{4} \exp \left(-\frac{2 L_{\mathcal{G}}^{2} T^{2}}{\gamma^{2}}\right) \int_{0}^{T} \int_{\Omega}\left|\frac{\partial}{\partial t}\left(z_{1}-z_{2}\right)\right|^{2}(x, t) d x d t \\
& +\frac{1}{4} \int_{0}^{T} \exp \left(-\frac{2 L_{\mathcal{G}}^{2} t^{2}}{\gamma^{2}}\right) \int_{\Omega}\left|\frac{\partial}{\partial t}\left(z_{1}-z_{2}\right)\right|^{2}(x, t) d x d t
\end{aligned}
$$

which in turn gives

$$
\left\|J\left(z_{1}\right)-J\left(z_{2}\right)\left|\left\|^{2} \leq \frac{1}{3}\right\|\right| z_{1}-z_{2} \mid\right\|^{2} .
$$

Hence $J$ is a contraction on the closed subset $B$ of $H^{1}(0, T ; V)$, which yields the existence and uniqueness of solutions.

At this point $(2.9)$ and the fact that $H^{1}\left(0, T ; L^{2}(\Omega)\right) \subset L^{2}\left(\Omega ; \mathcal{C}^{0}([0, T])\right)$ with continuous injection, entail that $\overline{\mathcal{G}}(u) \in L^{2}\left(\Omega ; \mathcal{C}^{0}([0, T])\right)$. Let us show that actually $\overline{\mathcal{G}}(u) \in H^{1}\left(0, T ; L^{2}(\Omega)\right)$.

It is not difficult to see that $(2.9)$, together with the fact that $\overline{\mathcal{G}}$ is a hysteresis operator, yields the following property for $\overline{\mathcal{G}}$, named piecewise Lipschitz continuity property

$$
\left\{\begin{array}{l}
\forall v \in \mathcal{M}\left(\Omega ; \mathcal{C}^{0}([0, T])\right), \forall\left[t_{1}, t_{2}\right] \subset[0, T] \\
\text { if } v(x, \cdot) \text { is affine in }\left[t_{1}, t_{2}\right] \text { a.e. in } \Omega \text {, then } \\
\left|[\overline{\mathcal{G}}(v)]\left(x, t_{2}\right)-[\overline{\mathcal{G}}(v)]\left(x, t_{1}\right)\right| \leq L_{\mathcal{G}}\left|v\left(x, t_{1}\right)-v\left(x, t_{2}\right)\right| \quad \text { a.e. in } \Omega .
\end{array}\right.
$$

As the family of continuous, piecewise linear functions is dense in $W^{1,1}(0, T)$, then (4.19) entails that $\overline{\mathcal{G}}(u) \in \mathcal{M}\left(\Omega ; W^{1,1}(0, T)\right)$, for all $u \in \mathcal{M}\left(\Omega ; W^{1,1}(0, T)\right)$; moreover

$$
\left|\frac{\partial}{\partial t} \overline{\mathcal{G}}(u)\right| \leq L\left|\frac{\partial u}{\partial t}\right| \quad \text { a.e. in } \Omega_{T} \text {. }
$$


But we have that $u \in H^{1}\left(0, T ; L^{2}(\Omega)\right)$; therefore from (4.20) we also deduce that $\overline{\mathcal{G}}(u) \in H^{1}\left(0, T ; L^{2}(\Omega)\right)$. This finishes the proof.

Remark 4.3. The proof that $\overline{\mathcal{G}}(u) \in H^{1}\left(0, T ; L^{2}(\Omega)\right)$ is the only point in the paper where the rate independence property of $\overline{\mathcal{G}}$ is used. For all the other results $\overline{\mathcal{G}}$ is not required to be also rate independent.

\subsection{Lipschitz continuous dependence on the data}

Theorem 4.4. (Lipschitz continuous dependence on the data).

Let $\overline{\mathcal{G}}: \mathcal{M}\left(\Omega ; \mathcal{C}^{0}([0, T])\right) \rightarrow \mathcal{M}\left(\Omega ; \mathcal{C}^{0}([0, T])\right)$ be the operator introduced in $(2.8)$. Then the dependence of the solution on the data is Lipschitz continuous in the following sense. For $i=1,2$, let $u_{i}^{0}, v_{i}^{0}, f_{i}$ be such that

$$
u_{i}^{0} \in V, \quad v_{i}^{0} \in H, \quad f_{i} \in L^{2}(0, T ; H) \quad i=1,2
$$

and let $\left(u_{i}, v_{i}\right)$ be the corresponding unique solution of Problem 4.1. Then

$$
\left\|u_{1}-u_{2}\right\|_{L^{2}\left(\Omega_{T}\right)}^{2} \leq c_{1}\left\|u_{1}^{0}-u_{2}^{0}\right\|_{L^{2}(\Omega)}^{2}+c_{2}\left\|v_{1}^{0}-v_{2}^{0}\right\|_{H}^{2}+c_{3}\left\|A^{-1}\left(f_{1}-f_{2}\right)\right\|_{L^{2}\left(\Omega_{T}\right)}^{2},
$$

where $c_{1} \equiv c_{1}\left(\gamma, T, L_{\mathcal{G}}\right), c_{2} \equiv c_{2}\left(\epsilon, \gamma, T, L_{\mathcal{G}}\right)$ and $c_{3} \equiv c_{3}\left(\sigma, \gamma, T, L_{\mathcal{G}}\right)$.

Proof. From our assumptions, we immediately have that the following system

$$
\left\{\begin{array}{l}
\epsilon A^{-1} \frac{\partial}{\partial t}\left(v_{1}-v_{2}\right)+\sigma A^{-1} \frac{\partial}{\partial t}\left(u_{1}-u_{2}\right)+\gamma\left(v_{1}-v_{2}\right)+\overline{\mathcal{G}}\left(u_{1}\right)-\overline{\mathcal{G}}\left(u_{2}\right)=\sigma A^{-1}\left(f_{1}-f_{2}\right) \\
\frac{\partial}{\partial t}\left(u_{1}-u_{2}\right)=v_{1}-v_{2},
\end{array}\right.
$$

holds in $L^{2}\left(\Omega_{T}\right)$. We multiply the first equation of the previous system by $v_{1}-v_{2}=$ $\frac{\partial}{\partial t}\left(u_{1}-u_{2}\right)$ in the scalar product of $L^{2}(\Omega)$. We get

$$
\begin{aligned}
\epsilon \frac{d D}{d t}(t) & +\frac{5}{8} \gamma\left\|\frac{\partial}{\partial t}\left(u_{1}-u_{2}\right)\right\|_{L^{2}(\Omega)}^{2}(t) \leq \frac{1}{\gamma}\left\|\overline{\mathcal{G}}\left(u_{1}\right)-\overline{\mathcal{G}}\left(u_{2}\right)\right\|_{L^{2}(\Omega)}^{2}(t) \\
& +\frac{2}{\gamma} \sigma^{2}\left\|A^{-1}\left(f_{1}-f_{2}\right)\right\|_{L^{2}(\Omega)}^{2}(t),
\end{aligned}
$$

where we recall that $D(t)=\frac{1}{2}\left\|v_{1}-v_{2}\right\|_{H}^{2}(t)$.

Working as we did in order to obtain (4.17), we deduce

$$
\begin{aligned}
& \frac{\epsilon}{2 \gamma} \frac{d D}{d t}(t)+\frac{5}{16}\left\|\frac{\partial}{\partial t}\left(u_{1}-u_{2}\right)\right\|_{L^{2}(\Omega)}^{2}(t) \leq \frac{L_{\mathcal{G}}^{2}}{\gamma^{2}}\left\|u_{1}(x, 0)-u_{2}(x, 0)\right\|_{L^{2}(\Omega)}^{2} \\
& +\frac{L_{\mathcal{G}}^{2} t}{\gamma^{2}} \int_{0}^{t} \int_{\Omega}\left|\frac{\partial}{\partial \tau}\left(u_{1}-u_{2}\right)\right|^{2}(x, \tau) d x d \tau+\frac{\sigma^{2}}{\gamma^{2}}\left\|A^{-1}\left(f_{1}-f_{2}\right)\right\|_{L^{2}(\Omega)}^{2}(t)
\end{aligned}
$$


Now we multiply (4.21) by $\exp \left(-\frac{2 L_{\mathcal{G}}^{2} t^{2}}{\gamma^{2}}\right)$ and we integrate in time from 0 to $T$. We denote by $\||\cdot|||$ the equivalent norm on $H^{1}\left(0, T ; L^{2}(\Omega)\right)$ which has been introduced in (4.18). We have

$$
\begin{aligned}
\left\|\left|u_{1}-u_{2}\left\|\left.\right|^{2} \leq \tilde{c}_{1}\right\| u_{1}(x, 0)-u_{2}(x, 0) \|_{L^{2}(\Omega)}^{2}\right.\right. & +\tilde{c}_{2}\left\|v_{1}(x, 0)-v_{2}(x, 0)\right\|_{H}^{2} \\
& +\tilde{c}_{3}\left\|A^{-1}\left(f_{1}-f_{2}\right)\right\|_{L^{2}\left(\Omega_{T}\right)}^{2},
\end{aligned}
$$

where $\tilde{c}_{1} \equiv \tilde{c}_{1}\left(\gamma, T, L_{\mathcal{G}}\right), \tilde{c}_{2} \equiv \tilde{c}_{2}(\epsilon, \gamma)$ and $\tilde{c}_{3} \equiv \tilde{c}_{3}(\sigma, \gamma)$. From this we finally deduce that

$$
\begin{aligned}
\left\|u_{1}-u_{2}\right\|_{L^{2}\left(\Omega_{T}\right)}^{2} \leq c_{1}\left\|u_{1}(x, 0)-u_{2}(x, 0)\right\|_{L^{2}(\Omega)}^{2} & +c_{2}\left\|v_{1}(x, 0)-v_{2}(x, 0)\right\|_{H}^{2} \\
& +c_{3}\left\|A^{-1}\left(f_{1}-f_{2}\right)\right\|_{L^{2}\left(\Omega_{T}\right)}^{2}
\end{aligned}
$$

with $c_{1} \equiv c_{1}\left(\gamma, T, L_{\mathcal{G}}\right), c_{2} \equiv c_{2}\left(\epsilon, \gamma, T, L_{\mathcal{G}}\right)$ and $c_{3} \equiv c_{3}\left(\sigma, \gamma, T, L_{\mathcal{G}}\right)$. This finishes the proof.

\subsection{Some further regularity results}

Theorem 4.5. (Regularity).

Let $\overline{\mathcal{G}}: \mathcal{M}\left(\Omega ; \mathcal{C}^{0}([0, T])\right) \rightarrow \mathcal{M}\left(\Omega ; \mathcal{C}^{0}([0, T])\right)$ be the operator introduced in (2.8) and let $f \in L^{2}\left(0, T ; H^{-1}(\Omega)\right), u^{0}, v^{0} \in H^{1}(\Omega)$. Then Problem 4.1 has a unique solution

$$
u \in H^{1}\left(0, T ; H^{1}(\Omega)\right), \quad v \in L^{2}\left(0, T ; H^{1}(\Omega)\right) .
$$

If instead $f \in L^{2}\left(0, T ; H^{-1}(\Omega)\right), u^{0}, v^{0} \in H_{0}^{1}(\Omega)$, then the unique solution of Problem 4.1 is such that

$$
u \in H^{1}\left(0, T ; H_{0}^{1}(\Omega)\right), \quad v \in L^{2}\left(0, T ; H_{0}^{1}(\Omega)\right) .
$$

In both cases it also turns out that

$$
v \in \mathcal{C}^{0}\left(0, T ; L^{2}(\Omega)\right)
$$

and there exists a constant $C>0$ such that

$$
\sup _{t \in[0, T]}\|v(t)\|_{L^{2}(\Omega)} \leq C\left(\|v\|_{L^{2}\left(0, T ; H^{1}(\Omega)\right)}+\left\|\frac{d v}{d t}\right\|_{L^{2}\left(0, T ; H^{-1}(\Omega)\right)}\right) .
$$

Proof. The proof of the first part of Theorem 4.5 is based on the classical characterization of the Sobolev spaces $H^{1}(\Omega)$ and $H_{0}^{1}(\Omega)$, which can be found for example in [2], Sections IX.1, IX.4.

Let us fix some notations. We consider any $D \subset \subset \Omega$, i.e. $D$ is an open bounded set contained in $\Omega$ such that $\bar{D} \subset \Omega$. Let $\chi_{D}$ be the characteristic function of the open set $D$; for any $h \in \mathbb{R}^{N}$ with $|h|<\operatorname{dist}\left(D, \Omega^{c}\right)$ we set

$$
\tau_{h} u(x):=u(x+h) \quad \text { and } \quad \delta_{h} u(x):=\tau_{h} u(x)-u(x) .
$$


Moreover let $(u, v)$ be the unique solution of Problem 4.1. We take the space increments in equation

$$
A^{-1} \frac{\partial}{\partial t}(\epsilon v+\sigma u)+\gamma v+\overline{\mathcal{G}}(u)=\sigma A^{-1} f
$$

which holds in $L^{2}\left(\Omega_{T}\right)$, where $v=\frac{\partial u}{\partial t}$; we multiply the result first by $\chi_{D}$ (so that now the equation holds in $\left.L^{2}(D \times(0, T))\right)$ and then by $\delta_{h} v \chi_{D}$ in the scalar product of $L^{2}(\Omega)$ (or equivalently in the scalar product of $L^{2}(D)$ ). Then we integrate in time between 0 and $T$. We get

$$
\begin{aligned}
& \int_{0}^{T} \int_{D}\left[A^{-1} \frac{\partial}{\partial t}\left(\epsilon \delta_{h} v+\sigma \delta_{h} u\right)\right]\left(\delta_{h} v\right) d x d t+\int_{0}^{T} \int_{D}\left(\gamma \delta_{h} v+\delta_{h} \overline{\mathcal{G}}(u)\right)\left(\delta_{h} v\right) d x d t \\
= & \frac{\epsilon}{2} \int_{0}^{T} \frac{\partial}{\partial t}\left\|\delta_{h} v\right\|_{H^{-1}(D)}^{2} d t+\int_{0}^{T}\left[\sigma\left\|\delta_{h} v\right\|_{H^{-1}(D)}^{2}+\gamma\left\|\delta_{h} v\right\|_{L^{2}(D)}^{2}\right] d t \\
& +\int_{0}^{T} \int_{D} \delta_{h} \overline{\mathcal{G}}(u) \delta_{h} v d x d t=\int_{0}^{T} \int_{D}\left(A^{-1} \delta_{h} f\right)\left(\delta_{h} v\right) d x d t \\
\leq & \frac{\gamma}{8} \int_{0}^{T}\left\|\delta_{h} v\right\|_{L^{2}(D)}^{2} d t+\frac{2}{\gamma} \sigma^{2} \int_{0}^{T}\left\|A^{-1} \delta_{h} f\right\|_{L^{2}(D)}^{2} d t .
\end{aligned}
$$

At this point, using assumption (2.10) and working as in (4.17)

$$
\begin{aligned}
\left\|\delta_{h} \overline{\mathcal{G}}(u)\right\|_{L^{2}\left(0, T ; L^{2}(D)\right)} & \leq\left\|\delta_{h} \overline{\mathcal{G}}(u)\right\|_{L^{2}\left(D ; \mathcal{C}^{0}([0, T])\right)} \\
& \leq L_{\mathcal{G}}\left\|\delta_{h} u\right\|_{L^{2}\left(D ; \mathcal{C}^{0}([0, T])\right)} \\
& \leq \sqrt{T} L_{\mathcal{G}}\left\|\delta_{h} v\right\|_{L^{2}\left(0, T ; L^{2}(D)\right)}+L_{\mathcal{G}}\left\|\delta_{h} u(x, 0)\right\|_{L^{2}(D)}
\end{aligned}
$$

from what we deduce

$$
\begin{aligned}
& \frac{\epsilon}{2}\left\|\delta_{h} v(x, T)\right\|_{H^{-1}(D)}^{2}+\sigma\left\|\delta_{h} v\right\|_{L^{2}\left(0, T ; H^{-1}(D)\right)}^{2} d t+\frac{7}{8} \gamma\left\|\delta_{h} v\right\|_{L^{2}\left(0, T ; L^{2}(D)\right)}^{2} \\
\leq & \frac{\gamma}{4}\left\|\delta_{h} v\right\|_{L^{2}\left(0, T ; L^{2}(D)\right)}^{2}+\frac{2}{\gamma} \sigma^{2}\left\|A^{-1} \delta_{h} f\right\|_{L^{2}\left(0, T ; L^{2}(D)\right)}^{2}+\frac{1}{\gamma}\left\|\delta_{h} \overline{\mathcal{G}}(u)\right\|_{L^{2}\left(0, T ; L^{2}(D)\right)}^{2} \\
& +\frac{\epsilon}{2}\left\|\delta_{h} v(x, 0)\right\|_{H^{-1}(D)}^{2} \leq\left(\frac{\gamma}{4}+2 \frac{T L_{\mathcal{G}}^{2}}{\gamma}\right)\left\|\delta_{h} v\right\|_{L^{2}\left(0, T ; L^{2}(D)\right)}^{2} \\
& +\frac{2}{\gamma} \sigma^{2}\left\|A^{-1} \delta_{h} f\right\|_{L^{2}\left(0, T ; L^{2}(D)\right)}^{2}+\frac{\epsilon}{2}\left\|\delta_{h} v(x, 0)\right\|_{H^{-1}(D)}^{2}+\frac{2}{\gamma} L_{\mathcal{G}}^{2}\left\|\delta_{h} u(x, 0)\right\|_{L^{2}(D)}^{2} .
\end{aligned}
$$

Using the same argument employed in the previous cases and exploiting our assumptions on the data, we obtain

$$
\frac{\epsilon}{2}\left\|\delta_{h} v(x, T)\right\|_{H^{-1}(D)}^{2}+\frac{\gamma}{8}\left\|\delta_{h} v\right\|_{L^{2}\left(0, T ; L^{2}(D)\right)}^{2} \leq C\left(T, \epsilon, \sigma, \gamma, L_{\mathcal{G}}\right)|h|^{2},
$$

where the constant $C$ depends on $T, \epsilon, \sigma, \gamma, L_{\mathcal{G}}$ but it is independent of $D$. Thus, using the characterization of the space $H^{1}(\Omega)$ we have that

$$
v(x, t) \in H^{1}(\Omega) \quad \text { a.e. in }[0, T]
$$


on the other hand, using the Lebesgue dominated convergence theorem, we also immediately get

$$
v \in L^{2}\left(0, T ; H^{1}(\Omega)\right) .
$$

which gives us the regularity we were looking for.

The case of the space $H_{0}^{1}(\Omega)$ can be carried on in a similar way.

Finally the last regularity results (4.22) and (4.23) are directly obtained using a classical theorem (see for example [23], pag. 191).

\subsection{Dependence of the solution on the parameters $\epsilon$ and $\sigma$}

We are able to state and prove the following two results

Theorem 4.6. (dependence on $\epsilon$ ).

For any given $\epsilon>0$, let $\left(u_{\epsilon}, v_{\epsilon}\right)$ be the unique solution of Problem 4.1 corresponding to the data

$$
f_{\epsilon}:=f \in L^{2}\left(0, T ; H^{-1}(\Omega)\right), \quad u_{\epsilon}^{0}:=u^{0} \in H_{0}^{1}(\Omega), \quad v_{\epsilon}^{0}:=v^{0} \in H_{0}^{1}(\Omega)
$$

such that

$$
u_{\epsilon} \in H^{1}\left(0, T ; H_{0}^{1}(\Omega)\right) \quad v_{\epsilon} \in L^{2}\left(0, T ; H_{0}^{1}(\Omega)\right) .
$$

Then there exist two functions $u \in H^{1}\left(0, T ; H_{0}^{1}(\Omega)\right)$ and $v \in L^{2}\left(0, T ; H_{0}^{1}(\Omega)\right)$ such that, if $\epsilon \rightarrow 0$, then

$$
\begin{array}{ll}
u_{\epsilon} \rightarrow u & \left\{\begin{array}{l}
\text { weakly in } H^{1}\left(0, T ; H_{0}^{1}(\Omega)\right) \\
\text { strongly in } L^{2}\left(\Omega ; \mathcal{C}^{0}([0, T])\right)
\end{array}\right. \\
v_{\epsilon} \rightarrow v & \text { weakly in } L^{2}\left(0, T ; H_{0}^{1}(\Omega)\right) ;
\end{array}
$$

moreover the pair $(u, v)$ fulfills the following system

$$
\left\{\begin{array}{l}
\sigma v-\triangle(\gamma v+\overline{\mathcal{G}}(u))=\sigma f \\
\frac{\partial u}{\partial t}=v
\end{array}\right.
$$

Proof. From our assumptions we obtain

$$
\left\{\begin{array}{l}
\epsilon \frac{\partial v_{\epsilon}}{\partial t}+\sigma v_{\epsilon}-\triangle\left(\gamma \frac{\partial u_{\epsilon}}{\partial t}+\overline{\mathcal{G}}\left(u_{\epsilon}\right)\right)=\sigma f \\
\frac{\partial u_{\epsilon}}{\partial t}=v_{\epsilon}
\end{array}\right.
$$

where the first equation of the previous system holds in $L^{2}\left(0, T ; H^{-1}(\Omega)\right)$. We test this equation by $\frac{\partial u_{\epsilon}}{\partial t}$, obtaining

$$
\begin{aligned}
& \frac{\epsilon}{2} \frac{d}{d t} \int_{\Omega}\left|\frac{\partial u_{\epsilon}}{\partial t}\right|^{2}(t) d x+\sigma \int_{\Omega}\left|\frac{\partial u_{\epsilon}}{\partial t}\right|^{2}(t) d x+\gamma \int_{\Omega}\left|\nabla\left(\frac{\partial u_{\epsilon}}{\partial t}\right)\right|^{2}(t) d x \\
\leq & \left.\frac{2 \sigma^{2}}{\gamma}|| f(t)\right|_{H^{-1}(\Omega)} ^{2}+\frac{\gamma}{8} \int_{\Omega}\left|\nabla\left(\frac{\partial u_{\epsilon}}{\partial t}\right)\right|^{2}(t) d x+\int_{\Omega}\left|\nabla \overline{\mathcal{G}}\left(u_{\epsilon}\right)(t)\right|\left|\nabla\left(\frac{\partial u_{\epsilon}}{\partial t}\right)(t)\right| d x .
\end{aligned}
$$


At this point, (2.10) yields, for any $t \in[0, T]$

$$
\begin{aligned}
& \left\|\nabla \overline{\mathcal{G}}\left(u_{\epsilon}\right)\right\|_{L^{2}(\Omega)}^{2} \leq\left\|\nabla \overline{\mathcal{G}}\left(u_{\epsilon}\right)\right\|_{L^{2}\left(\Omega ; \mathcal{C}^{0}([0, T])\right)}^{2} \leq L_{\mathcal{G}}^{2}\left\|\nabla u_{\epsilon}\right\|_{L^{2}\left(\Omega ; \mathcal{C}^{0}([0, T])\right)}^{2} \\
\leq & 2 L_{\mathcal{G}}^{2}\left\|\nabla u^{0}\right\|_{L^{2}(\Omega)}^{2}+2 L_{\mathcal{G}}^{2} t \int_{\Omega} \int_{0}^{t}\left|\nabla\left(\frac{\partial u_{\epsilon}}{\partial t}\right)\right|^{2} d t d x .
\end{aligned}
$$

This in turn entails that

$$
\begin{aligned}
& \frac{\epsilon}{4 \gamma} \frac{d}{d t} \int_{\Omega}\left|\frac{\partial u_{\epsilon}}{\partial t}\right|^{2}(t) d x+\frac{5}{16} \int_{\Omega}\left|\nabla\left(\frac{\partial u_{\epsilon}}{\partial t}\right)\right|^{2}(t) d x \\
\leq & \frac{\sigma^{2}}{\gamma^{2}}\|f(t)\|_{H^{-1}(\Omega)}^{2}+\frac{1}{2 \gamma^{2}}\left\|\nabla \overline{\mathcal{G}}\left(u_{\epsilon}\right)(t)\right\|_{L^{2}(\Omega)}^{2} \\
\leq & \frac{\sigma^{2}}{\gamma^{2}}\|f(t)\|_{H^{-1}(\Omega)}^{2}+\frac{L_{\mathcal{G}}^{2}}{\gamma^{2}}\left\|\nabla u^{0}\right\|_{L^{2}(\Omega)}^{2}+\frac{L_{\mathcal{G}}^{2} t}{\gamma^{2}} \int_{\Omega} \int_{0}^{t}\left|\nabla\left(\frac{\partial u_{\epsilon}}{\partial t}\right)\right|^{2} d t d x .
\end{aligned}
$$

We are now basically in the same situation as in (4.21). We use therefore the same idea of multiplying $(4.25)$ by $\exp \left(-\frac{2 L_{\mathcal{G}}^{2} t^{2}}{\gamma^{2}}\right)$ and integrating in time for $t \in(0, T)$; we use once more the equivalent norm \|\|$\cdot\|\|$ introduced in (4.18). We obtain

$$
\begin{aligned}
\frac{5}{16}\|\| \nabla u_{\epsilon}\|\|^{2} \leq \frac{\epsilon}{4 \gamma}\left\|v^{0}\right\|_{L^{2}(\Omega)}^{2} & +\tilde{c}\left\|\nabla u^{0}\right\|_{L^{2}(\Omega)}^{2}+\frac{1}{4}\left\|\nabla u_{\epsilon}\right\| \|^{2} \\
& +\frac{\sigma^{2}}{\gamma^{2}} \int_{0}^{T} \exp \left(-\frac{2 L_{\mathcal{G}}^{2} t^{2}}{\gamma^{2}}\right)\|f(t)\|_{H^{-1}(\Omega)}^{2} d t,
\end{aligned}
$$

where $\tilde{c}$ only depends on $L_{\mathcal{G}}, \gamma, T$ and it is independent of $\epsilon$ and $\sigma$.

Now, as at the end we will consider $\epsilon \rightarrow 0$, it is not restrictive to assume $\epsilon<1$. This allows us to obtain the following estimate

$$
\left\|u_{\epsilon}\right\|_{H^{1}\left(0, T ; H_{0}^{1}(\Omega)\right)} \leq \text { constant (independent of } \epsilon \text { ). }
$$

Standard interpolation results then yield the desired passage to the limit.

Remark 4.7. For slow processes the displacement term $\epsilon \frac{\partial^{2} \vec{B}}{\partial t^{2}}$ in (3.4) is dominated by the Ohmic term $4 \pi \sigma \frac{\partial \vec{B}}{\partial t}$. In this case (3.4) is then usually replaced by the so called eddy-current equation

$$
4 \pi \sigma \frac{\partial \vec{B}}{\partial t}+c^{2} \nabla \times \nabla \times \vec{H}=4 \pi c \sigma \nabla \times \vec{g} \quad \text { in } Q_{T} .
$$

If now we apply to the fields $\vec{B}$ and $\vec{H}$ in (4.27) the same restrictions as we did in Section 3 and couple the result with the constitutive relation (3.6), we obtain (4.24).

Theorem 4.8. (dependence on $\sigma$ ).

For any given $\sigma>0$, let $\left(u_{\sigma}, v_{\sigma}\right)$ be the unique solution of Problem 4.1 corresponding to the data

$$
f_{\sigma}:=f \in L^{2}\left(0, T ; H^{-1}(\Omega)\right), \quad u_{\sigma}^{0}:=u^{0} \in H_{0}^{1}(\Omega), \quad v_{\sigma}^{0}:=v^{0} \in H_{0}^{1}(\Omega)
$$


such that

$$
u_{\sigma} \in H^{1}\left(0, T ; H_{0}^{1}(\Omega)\right) \quad v_{\sigma} \in L^{2}\left(0, T ; H_{0}^{1}(\Omega)\right) .
$$

Then there exist two functions $u \in H^{1}\left(0, T ; H_{0}^{1}(\Omega)\right)$ and $v \in L^{2}\left(0, T ; H_{0}^{1}(\Omega)\right)$ such that, if $\sigma \rightarrow 0$, then

$$
\begin{array}{ll}
u_{\sigma} \rightarrow u & \left\{\begin{array}{l}
\text { weakly in } H^{1}\left(0, T ; H_{0}^{1}(\Omega)\right) \\
\text { strongly in } L^{2}\left(\Omega ; \mathcal{C}^{0}([0, T])\right)
\end{array}\right. \\
v_{\sigma} \rightarrow v & \text { weakly in } L^{2}\left(0, T ; H_{0}^{1}(\Omega)\right) ;
\end{array}
$$

moreover the pair $(u, v)$ satisfies the following system

$$
\left\{\begin{array}{l}
\epsilon \frac{\partial v}{\partial t}-\Delta(\gamma v+\overline{\mathcal{G}}(u))=0 \\
\frac{\partial u}{\partial t}=v
\end{array}\right.
$$

Proof. The proof of this theorem relays on the same idea employed in the proof of Theorem 4.6. Indeed, as this time we are going to take $\sigma \rightarrow 0$, then it is not restrictive to assume $\sigma<1$. Therefore (4.26) leads to the following estimate

$$
\left\|u_{\sigma}\right\|_{H^{1}\left(0, T ; H_{0}^{1}(\Omega)\right)} \leq \text { constant (independent of } \sigma \text { ). }
$$

The desired passage to the limit is now carried on by means of some standard arguments.

Remark 4.9. Equation (3.4) applies for both ferromagnetic and ferrimagnetic materials. The case $\sigma=0$ in (3.4) corresponds to ferrimagnetic insulators. In this case $\vec{J}=0$ in the whole $\mathbb{R}^{3}$ and (3.4) becomes

$$
\epsilon \frac{\partial^{2} \vec{B}}{\partial t^{2}}+c^{2} \nabla \times \nabla \times \vec{H}=0 \quad \text { in } Q_{T},
$$

where we account for the displacement currents. This equation applies for either slow or fast processes. Also in this case, suitable restrictions on the fields involved lead to (4.28), where (3.6) is once more used. We refer to [27] and [28] for more details concerning the discussion of the vectorial models (4.27) and (4.29).

\section{A more realistic physical situation}

We know that by coupling in a suitable way the Maxwell equations and the Ohm law, we are able to describe electromagnetic processes in ferromagnetic materials. In Section 3 we have chosen to work in a bounded domain $Q$ of $\mathbb{R}^{3}$ but this approach does not seem too realistic; in fact the evolution of the fields outside $Q$ may have an influence on the processes which take place inside $Q$ and it seems difficult to describe in a proper way this interaction by formulating a boundary value problem on a bounded domain. 
In a recent paper (see [28]), Visintin considers the Maxwell equations and the Ohm law set in the whole Euclidean space $\mathbb{R}^{3}$ and relates the magnetic induction $\vec{B}$ and the magnetic field $\vec{H}$ by means of two constitutive relations, inside and outside a prescribed bounded domain; then he carries on his analysis working in the whole $\mathbb{R}^{3}$. Here we would like to follow the same idea.

Consider an electromagnetic process in a magnetic material which occupies a Euclidean domain $Q \subset \mathbb{R}^{3}$ in a time interval $(0, T)$. We set for the sake of simplicity

$$
Q_{T}:=Q \times(0, T) \quad \mathbb{R}_{T}^{3}:=\mathbb{R}^{3} \times(0, T) .
$$

The Maxwell equations in the whole $\mathbb{R}^{3}$ (for the sake of simplicity we state only the ones which will be useful later) read

$$
\begin{array}{ll}
c \nabla \times \vec{H}=4 \pi \vec{J}+\frac{\partial \vec{D}}{\partial t} & \text { in } \mathbb{R}_{T}^{3} \\
c \nabla \times \vec{E}=-\frac{\partial \vec{B}}{\partial t} & \text { in } \mathbb{R}_{T}^{3}
\end{array}
$$

where $\vec{H}$ is the magnetic field, $\vec{B}$ is the magnetic induction, $\vec{E}$ is the electric field $\vec{J}$ is the electric current density, $\vec{D}$ is the electric displacement and finally $c$ is the speed of light in vacuum.

Now, suppose to deal with a ferromagnetic metal; in this setting $\vec{D}=\epsilon \vec{E}$, where $\epsilon$ is the electric permittivity; therefore we can rewrite (5.1) in the following way

$$
c \nabla \times \vec{H}=4 \pi \vec{J}+\epsilon \frac{\partial \vec{E}}{\partial t} \quad \text { in } \mathbb{R}_{T}^{3} .
$$

We denote by $\vec{E}_{\text {app }}$ a prescribed applied electromotive force (this is possible, for example, with a battery); we assume that the electric permittivity $\epsilon$ is a scalar constant and moreover we introduce the electric conductivity $\sigma$ which is assumed to vanish outside $Q$. Concerning the material we are dealing with, we suppose that it is surrounded by vacuum and that an electric current can circulate in an exterior conductor; we can see this assuming that $\vec{J}$ equals a prescribed time dependent vector field $\vec{J}_{\text {ext }}$ which vanishes inside $Q$.

After this preamble (3.3) can be extended to the following law

$$
\vec{J}= \begin{cases}\sigma\left(\vec{E}+\vec{E}_{\mathrm{app}}\right) & \text { in } Q \times(0, T) \\ \vec{J}_{\mathrm{ext}} & \text { in }\left[\mathbb{R}^{3} \backslash Q\right] \times(0, T) .\end{cases}
$$

Moreover, after the assumptions we made on the conductivity, $\sigma$ can be accordingly replaced by $\chi_{Q}$, the characteristic function of the set $Q$, i.e. $\chi_{Q}=1$ inside $Q$ and $\chi_{Q}=0$ outside $Q$. Thus (5.3) becomes

$$
\vec{J}=\chi_{Q}\left(\vec{E}+\vec{E}_{\mathrm{app}}\right)+\left(1-\chi_{Q}\right) \vec{J}_{\mathrm{ext}} \quad \text { in } \mathbb{R}_{T}^{3}
$$


and we come at the end to the following system

$$
\begin{cases}c \nabla \times \vec{H}=4 \pi\left[\chi_{Q}\left(\vec{E}+\vec{E}_{\mathrm{app}}\right)+\left(1-\chi_{Q}\right) \vec{J}_{\mathrm{ext}}\right]+\epsilon \frac{\partial \vec{E}}{\partial t} & \text { in } \mathbb{R}_{T}^{3} . \\ c \nabla \times \vec{E}=-\frac{\partial \vec{B}}{\partial t} & \end{cases}
$$

This vectorial model must be coupled with a suitable constitutive relation. We could choose for example to relate $\vec{B}$ and $\vec{H}$ by means of a constitutive law with hysteresis inside $Q$ and simply to set $\vec{B}=\vec{H}$ outside $Q$. This could be made explicit in the following way

$$
\vec{H}=\chi_{Q}\left(\mathcal{F}(\vec{B})+\gamma \frac{\partial \vec{B}}{\partial t}\right)+\left(1-\chi_{Q}\right) \vec{B},
$$

where $\mathcal{F}$ is a suitable vector hysteresis operator and $\gamma>0$ is a nonnegative relaxation parameter. Therefore the model we would like to study is at the end the following

$$
\begin{cases}c \nabla \times \vec{H}=4 \pi\left[\chi_{Q}\left(\vec{E}+\vec{E}_{\mathrm{app}}\right)+\left(1-\chi_{Q}\right) \vec{J}_{\mathrm{ext}}\right]+\epsilon \frac{\partial \vec{E}}{\partial t} & \\ c \nabla \times \vec{E}=-\frac{\partial \vec{B}}{\partial t} & \text { in } \mathbb{R}_{T}^{3} . \\ \vec{H}=\chi_{Q}\left(\mathcal{F}(\vec{B})+\gamma \frac{\partial \vec{B}}{\partial t}\right)+\left(1-\chi_{Q}\right) \vec{B} & \end{cases}
$$

The problem of finding well posedness results for this model is for the moment an open question. However also in this case some restrictions on the fields involved can be assumed and solutions to (5.4) in some particular situations can be found. We refer to [11] for existence and uniqueness of a solution (corresponding to a plane wave) for a one-dimensional counterpart of (5.4).

Acknowledgement. The author wishes to acknowledge helpful and stimulating discussions with Proff. Martin Brokate, Pavel Krejčí and Augusto Visintin who certainly contributed to the final draft of the paper providing many useful suggestions and remarks. The author wishes also to thank the kind hospitality of the WIAS - Weierstrass Institute for Applied Analysis and Stochastics - and of the Technische Universität München (Zentrum Mathematik M6).

\section{References}

[1] G. Bertotti: Hysteresis in magnetism, Academic Press, Boston (1998).

[2] H. Brezis: Analyse fonctionnelle, Théorie et applications, Masson, Paris (1983).

[3] M. Brokate: Some BV properties of the Preisach hysteresis operator, Appl. Anal. 32, (1989), no. 3-4, 229-252. 
[4] M. Brokate: On a characterization of the Preisach model for hysteresis, Rend. Sem. Mat. Univ. Padova 83 (1990), 153-163.

[5] M. Brokate, J. Sprekels: Hysteresis and phase transitions, Applied Mathematical Sciences, 121, Springer-Verlag, New York (1996).

[6] M. Brokate, A. Visintin: Properties of the Preisach model for hysteresis, J. Reine Angew. Math., 402 (1989), 1-40.

[7] E. Della Torre: Magnetic hysteresis, I.E.E.E. Press (1999).

[8] M. Eleuteri: "Some P.D.E.s with hysteresis", Proceedings of the International Conference "Free Boundary Problems - Theory and Applications", (Coimbra, Portugal, 7-12 June 2005), Isabel Narra Figueiredo, José Francisco Rodrigues and Lisa Santos (Editors), to appear.

[9] M. Eleuteri: "On some P.D.E.s with hysteresis", Ph.D. Thesis, University of Trento (2006).

[10] M. Eleuteri, P. KREJČÍ: "Asymptotic convergence results for a system of partial differential equations with hysteresis arising in electromagnetism", submitted for publication.

[11] M. ELEUTERI: "On a model for electromagnetic processes in ferromagnetic materials with hysteresis and space dependent electric conductivity", in preparation.

[12] J.D. JaCKson: Classical electrodynamics, New York, Wiley 1975.

[13] M.A. Krasnosel'skiI , A.V. PokrovskiĬ: Systems with hysteresis, Springer, Berlin (1989), Russian edition: Nauka, Moscow (1983).

[14] P. KRejČÍ: On Maxwell equations with the Preisach hysteresis operator: the one-dimensional time-periodic case, Apl. Mat., 34 (1989), 364-374.

[15] P. KREJČÍ: Hysteresis, convexity and dissipation in hyperbolic equations, Tokyo: Gakkotosho (1996).

[16] P. KREJČÍ: Long-time behaviour of solutions to hyperbolic equations with hysteresis, Handbook of Evolution Equations: Evolutionary Equations, Vol. 2 (eds. C. M. Dafermos and E. Feireisl), Elsevier 2005, 303-370.

[17] P. Krejčí, Ph. Laurençot: Generalized variational inequalities, J. Convex Anal., 9 (2002), 159-183.

[18] I.D. Mayergoyz: Mathematical models of hysteresis, Springer, New York (1991).

[19] A. Mielke: Analysis of energetic models for rate-independent materials, Proceedings of the I.C.M., Vol. III (Beijing, 2002), Higher Ed. Press, Beijing (2002), 817-828. 
[20] A. Mielke, F. Theil: On rate-independent hysteresis models, NoDEA - Nonlinear Differential Equations Appl., 2 (2004), 151-189.

[21] A. Mielke, F. Theil, V. Levitas: A variational formulation of rateindependent phase transformations using an extremum principle, Arch. Rational Mech. Anal., 162 (2002), 137-177.

[22] F. Preisach: Über die magnetische Nachwirkung, Z. Physik, 94 (1935), 277302. (In German).

[23] J.C. Robinson: Infinite-dimensional dynamical systems. An introduction to dissipative parabolic PDEs and the theory of global attractors, Cambridge Texts in Applied Mathematics, Cambridge University Press, Cambridge (2001).

[24] L. Schwartz: Distributions à valeurs vectorielles, Parts I, II, Ann. Inst. Fourier 7 (1957) 1-141; 8 (1958), 1-209.

[25] A. Visintin: On the Preisach model for hysteresis, Nonliner Analysis, T.M.A. 9 (1984), 977-996.

[26] A. Visintin: Differential Models of Hysteresis, Springer (1994).

[27] A. Visintin: Vector Preisach model and Maxwell's equations, Physica B, 306 (2001), 21-25.

[28] A. Visintin: Maxwell's equations with vector hysteresis, Arch. Rational Mech. Anal., 175 (2005), 1-37. 